Revue internationale P.M.E.

Économie et gestion de la petite et moyenne entreprise

\title{
La performance des PMI dans l'attaque des marchés internationaux : une étude de cas dans un contexte d'île périphérique de la CEE
}

\section{Michel Boyer}

Volume 7, numéro 2, 1994

URI : https://id.erudit.org/iderudit/1008389ar

DOI : https://doi.org/10.7202/1008389ar

Aller au sommaire du numéro

Éditeur(s)

Presses de l’Université du Québec

ISSN

0776-5436 (imprimé)

1918-9699 (numérique)

Découvrir la revue

Citer cet article

Boyer, M. (1994). La performance des PMI dans l'attaque des marchés internationaux : une étude de cas dans un contexte d'île périphérique de la CEE. Revue internationale P.M.E., 7(2), 59-83. https://doi.org/10.7202/1008389ar
Résumé de l'article

Cette recherche s'inscrit dans une suite de travaux cherchant à préciser la relation entre la performance à l'exportation de la PMI et certaines de ses caractéristiques. Sa spécificité est de tenter de neutraliser l'incidence des facteurs environnementaux dans l'observation de cette relation.

L'analyse porte, pour cette raison, sur des cas d'entreprises relevant exclusivement de la même industrie et de la même localisation géographique : l'observation de situations de réussite et d'échec à l'exportation, influencées de la même façon par les variables d'environnement, facilite ainsi la comparaison des différents comportements stratégiques et opérationnels des firmes étudiées et fait ressortir un ensemble de facteurs de performance d'origine interne.

L'intervention d'experts, inspirée des principes de la méthode Delphi, aboutit à la hiérarchisation des facteurs explicatifs les plus importants et à leur classement en trois groupes : les facteurs de choix stratégique, les caractéristiques du dirigeant de la PMI et les facteurs liés au dispositif opérationnel de l'entreprise. L'analyse des résultats permet également de proposer quelques conclusions utiles aux décideurs en matière de promotion du développement international des PMI dans un contexte d’île périphérique de la CEE. 


\title{
La performance des PMI dans l'attaque des marchés internationaux: une étude de cas dans un contexte d'île périphérique de la CEE
}

\author{
Michel BOYER* \\ Université de la Réunion
}

\begin{abstract}
RÉSUMÉ
Cette recherche s'inscrit dans une suite de travaux cherchant à préciser la relation entre la performance à l'exportation de la PMl et certaines de ses caractéristiques. Sa spécificité est de tenter de neutraliser l'incidence des facteurs environnementaux dans l'observation de cette relation.

L'analyse porte, pour cette raison, sur des cas d'entreprises relevant exclusivement de la même industrie et de la même localisation géographique: l'observation de situations de réussite et d'échec à l'exportation, influencées de la même façon par les variables d'environnement, facilite ainsi la comparaison des différents comportements stratégiques et opérationnels des firmes étudiées et fait ressortir un ensemble de facteurs de performance d'origine interne.

L'intervention d'experts, inspirée des principes de la méthode Delphi, aboutit à la hiérarchisation des facteurs explicatifs les plus importants et à leur classement en trois groupes: les facteurs de choix stratégique, les caractéristiques du dirigeant de la PMI et les facteurs liés au dispositif opérationnel de l'entreprise. L'analyse des résultats permet également de proposer quelques conclusions utiles aux décideurs en matière de promotion du développement international des PMI dans un contexte d'île périphérique de la CEE.
\end{abstract}

* Michel Boyer est enseignant-chercheur en sciences de gestion à l'Université de la Réunion où il dirige l'Institut d'administration des entreprises. Membre du Groupe de recherche et d'étude sur la gestion et l'entreprise dans l'océan Indien (GREGEOI), il enseigne en finance et en stratégie. Ses recherches actuelles portent sur le développement des PME en situation d'insularité. L'auteur est titulaire d'un doctorat et d'une habilitation à diriger des recherches en sciences de gestion obtenus en France (IAE d'Aix-en-Provence). Adresse: Université de la Réunion, Institut d'administration des entreprises, B.P. 356, 97468 Saint-Denis, Cedex, France. 


\section{ABSTRACT}

This research work takes place within a series of studies endeavouring to clarify the relationship between the performance of Small and Medium size Industries (SMI) concerning exports and some of its characteristics. Specifically, the research attempts to neutralize the incidence of environmental factors when observing this relationship.

For this reason, this analysis looks exclusively at the case of firms within the same industry and situated in the same geographical area: the observation of situations of success and failure in exporting, influenced in the same way by environmental variables, facilitates thus a comparison of the different strategic and operational behaviours of these firms and underlines a set of internal factors of performance.

The intervention of experts, inspired by the principles of the Delphi method, results in the hierarchical ordering of the most important contributing factors and their classification into three groups: the factors of strategic choice, the characteristics of the manager of the SMI and the factors bound up with the operational structure of the firm. The analysis of the results also suggests several conclusions useful for decision-making concerning the promotion of international development of SMI in the context of an island on the periphery of $E E C$.

\section{RESUMEN}

Esta investigacion se inscribe dentro de una serie de trabajos que intentan aclarar cual es la relacion entre los resultados de la exportacion de las pequenas y medianas industrias y algunas de sus caracteristicas. Su caracter especifico reside en intentar neutralizar la incidencia de los factores circandantes en la observacion de dicha relacion.

Por eso, el analisis se concentra en los casos de las empresas que dependen exclusivamente de la misma industria y de la misma situacion geografica: la observacion de situaciones de éxito o fracaso con respecto a la exportacion, situaciones influenciadas asimismo por las variables del entorno, facilita la comparacion entre los diferentes comportamientos estratégicos y operacionales de las firmas estudiadas y destaca, a su vez, una serie de factores de origen interno que influyen en los resultados.

La intervencion de expertos, inspirada de los principios del método Delphi, desemboca en una jerarquizacion de los factores explicativos mas importantes y en una clasificacion en tres grupos: los factores de eleccion estratégica, las caracteristicas del dirigente de la PyMI y los factores ligados al dispositivo operacional de la empresa. El analisis de los resultados permite sacar conclusiones utiles para quienes deciden en la promocion del desarrollo internacional de las PyMI en el ambito de una isla periférica de la CEE.

\section{Introduction}

Il est devenu presque banal d'affirmer que l'exportation constitue une priorité pour la plupart des pays, en voie de développement ou développés, et qu'elle joue un rôle capital dans leur croissance. 
Quel que soit l'état de développement du pays exportateur, l'exportation est surtout le fait des entreprises de grande taille; cependant, les PMI représentent un potentiel considérable de création de richesse, comme en témoigne l'exemple allemand où elles jouent un rôle très actif dans les excédents commerciaux. Des dispositifs d'incitation sont ainsi mis en place en leur faveur par les pouvoirs publics, notamment en France (Baudoux et Roncin, 1980; Ricol, 1990).

L'identification scientifique des facteurs affectant le succès de la PME sur les marchés d'exportation est donc de la plus haute importance. Des observations faites entre 1988 et 1991 à l'île de la Réunion ont permis une contribution à cette recherche.

Située dans la partie sud-ouest de l'océan Indien, à 800 kilomètres à l'est de Madagascar, à 9000 kilomètres de Paris, cette île française, de $2512 \mathrm{~km}^{2}$, est peuplée de 600000 habitants. Son tissu économique est composé quasi exclusivement d'entreprises de petite ou moyenne dimension. D'après les critères énoncés - nombre d'employés et montant des actifs - par Julien et Marchesnay (1988), une dizaine d'opérateurs privés seulement peuvent être qualifiés de «grosses ou très grosses entreprises».

L'économie de cette île de l'océan Indien se caractérise également par le fait que le secteur non marchand - services fournis par les administrations et les collectivités locales à titre gratuit ou quasi gratuit - représente $30 \%$ du PIB, le secteur tertiaire marchand $47 \%$, le secteur primaire $5 \%$ et le secteur secondaire $18 \%$.

Un fort taux de chômage ( $38 \%$ ), un nombre exceptionnel de bénéficiaires du Revenu Minimum d'Insertion et de titulaires d'un Contrat Emploi-Solidarité couplé à un montant élevé des transferts publics et sociaux (13 milliards de francs pour un PIB de 23 milliards de francs en 1991) provenant de la France métropolitaine, confèrent à cette économie un caractère souvent qualifié d'artificiel.

Dans un tel contexte, toute activité génératrice de valeur ajoutée durable est recherchée et favorisée par les pouvoirs publics : de nombreuses incitations au développement du secteur productif ont conduit à l'émergence d'une industrie locale. Cette industrie réunionnaise se décompose en trois catégories : bâtiments et travaux publics (BTP), l'industrie sucrière et les autres PMI.

Cette troisième catégorie constitue le champ global d'investigation de notre recherche, les deux premières présentant des caractéristiques particulières (ancienneté de l'activité, marchés administrés, métier relativement fermé) qui ont très peu de points communs avec celles des autres PMI. 
Ces PMI, au nombre de 280 environ, relèvent principalement des secteurs de l'agro-alimentaire, des industries mécaniques et des matériaux de construction. Elles se caractérisent par leur jeunesse, $60 \%$ d'entre elles ayant moins de 10 ans. Elles concourent de plus en plus à la formation du PIB : $9 \%$ en 1991 contre $6 \%$ en 1983 et $3,6 \%$ en 1970 .

Cette évolution sectorielle, apparemment favorable, cache en fait des situations souvent très fragiles: certaines activités n'existent que par le maintien de marchés administrativement protégés par l'attribution de subventions de tous ordres ou par des mesures de réglementation spécifique (comme l'octroi de mer taxant les importations).

Il existe cependant quelques entreprises qui, ayant réussi leur implantation industrielle dans des activités d'import-substitution sur le marché local, tentent de générer une valeur ajoutée supplémentaire en s'attaquant à des marchés internationaux - le marché métropolitain en faisant partie, car la Réunion est elle-même considérée comme territoire d'exportation pour les entreprises françaises. Ces activités d'exportation ne sont encore qu'à l'état embryonnaire, mais certaines d'entre elles présentent des évolutions substantielles: c'est le cas des boissons spiritueuses (punchs et liqueurs exotiques) avec plus de $100 \%$ de taux de croissance des exportations en valeur entre 1989 et 1990, ainsi qu'entre 1990 et 1991. De tels taux s'expliquent:

- par le fait que les tentatives d'exportation de ces sous-produits de la canne sont toutes récentes, l'exploitation de la canne à sucre à la Réunion ayant été, depuis son origine, quasi exclusivement orientée vers la production de sucre roux en vrac destiné aux raffineries métropolitaines;

- par le faible nombre de producteurs dans ce secteur marqué par un mouvement continuel de restructurations depuis l'origine de la production de rhum dans l'île. Actuellement, il ne reste plus que trois PMI qui assurent la satisfaction des besoins du marché local en punchs et liqueurs exotiques et toutes trois se sont lancées récemment dans des expériences d'exportation.

L'analyse de formes similaires de développement international de PME a déjà été traitée dans de nombreuses recherches menées en France et dans le monde entier. Celles-ci peuvent être réparties en deux catégories:

1. Celles qui ont pour objet d'étudier et d'expliquer le comportement d'engagement des PMI dans l'activité internationale. Ainsi, des modèles explicatifs de la décision d'exporter en PMI ont été élaborés (Wiedersheim-Paul et al., 1978; Roux, 1991) et testés empiriquement. Roux (1991) étudie l'effet respectif des variables individuelles, d'envi- 
ronnement et d'entreprise sur la décision d'exporter. L'auteur montre que le fait qu'une entreprise décide d'exporter est largement expliqué par le profil individuel du dirigeant, décrit par son attitude vis-à-vis du risque, son stock de connaissances et son niveau d'éducation, son degré d'ouverture et d'exposition aux médias, ainsi que par son expérience préalable des cultures étrangères.

2. Celles qui ont pour objet d'étudier et d'expliquer les niveaux de performance atteints dans l'activité d'exportation, une fois prise la décision d'exporter - la performance étant mesurée ici par la part relative des exportations dans les ventes totales (Kleinschmidt et Ross, 1984 ; Aaby et Slater, 1989; McNaughton, 1992). Le travail de recherche consiste ainsi à analyser les raisons pour lesquelles certaines entreprises de petite ou moyenne dimension réussissent dans leurs activités internationales et d'autres, non. Deux catégories de facteurs sont ainsi identifiées: ceux qui caractérisent l'environnement de l'entreprise et ceux qui relèvent de son fonctionnement interne.

Notre travail se rapporte à cette deuxième catégorie, avec cependant une spécificité : nous avons voulu, à la différence des recherches précédentes, neutraliser l'influence des interactions entre variables d'environnement et variables d'entreprise sur l'explication de la performance. Aussi, notre champ d'étude ne comprend-il qu'une seule localisation géographique, la Réunion, et qu'une seule industrie, celle des spiritueux.

Nous cherchons ainsi à analyser plus clairement l'incidence des variables d'entreprise sur la performance à l'exportation et à mettre en évidence leur poids respectif dans l'explication de la réussite.

Pour cela, nous procéderons tout d'abord à l'identification d'un ensemble de facteurs issus de la littérature (première section), puis à l'étude d'expériences de développement international menées par les PMI réunionnaises productrices de spiritueux. La méthode utilisée est exposée en deuxième section; l'analyse des résultats obtenus (troisième section) permettra de tirer quelques conclusions (quatrième section) utiles à la rationalisation des efforts de promotion par les pouvoirs publics du développement international des PMI.

\section{Identification des facteurs favorisant la réussite des PMI exportatrices}

Un grand nombre de travaux, durant les vingt dernières années, ont traité de l'identification des facteurs favorisant la performance des entreprises à l'exportation. En France, les travaux de Baudoux et Roncin (1980; 1982), Reffait et 
Roux (1981), Giordano (1984; 1985), Joffre (1984; 1986), Pras et Roux (1985), Roux (1991) permettent de faire le point sur la nature des facteurs influençant favorablement le succès des PMI nationales exportatrices. Sur le plan international, Bilkey (1978) a fait un premier travail de synthèse des recherches sur la réussite à l'exportation, poursuivi et complété par ceux de Miesenbock (1988), de Aaby et Slater (1989).

Ces travaux facilitent considérablement la tâche du chercheur dans son souci de retenir une liste de base aussi exhaustive que possible de facteurs explicatifs de la performance de l'entreprise sur les marchés internationaux.

Néanmoins, pour construire cette liste théorique, nous avons besoin d'un référentiel permettant de catégoriser les divers facteurs mis en évidence dans la littérature.

\subsection{Cadre de référence}

Il n'existe pas encore, à l'heure actuelle, de modèle économétrique capable de décrire complètement la nature des liaisons existant entre la performance à l'exportation (notée PERF) et l'ensemble de ses variables explicatives. La plupart des travaux procèdent du test d'hypothèses par les méthodes classiques d'analyse de corrélation entre une variable expliquée (la mesure de performance) et une famille de variables explicatives potentielles. La difficulté majeure, puisqu'il s'agit d'entreprises, est de trouver un échantillon d'observations homogènes de taille suffisante pour pouvoir conclure à une relation précise et donner ses conditions de validité. Nous observons que les auteurs cités ci-dessus utilisent deux catégories de variables explicatives.

1. Celles qui décrivent l'environnement de l'entreprise (notées ENVT). Le terme «environnement» sert à désigner les facteurs macro-économiques, sociaux, techniques, culturels et politiques qui agissent sur le niveau de performance et sur lesquels l'entreprise n'a que très peu d'influence. Ces facteurs varient selon la localisation géographique de l'entreprise, le secteur (au sens de Porter, 1982) et les marchés concernés.

2. Celles relatives à la stratégie et au fonctionnement interne de l'entreprise (notées génériquement ENTR): ce sont des facteurs relevant des décisions de management prises et contrôlées par la direction de l'entreprise. Nous rajoutons dans cette catégorie les caractéristiques individuelles du chef d'entreprise afin de faire apparaître explicitement son rôle dans la performance, tout à fait capital dans le cas de PME (Johnston et Czinkota, 1982; Castaldi, 1986). 
Notre cadre de référence admet explicitement cette distinction, classique en stratégie, entre environnement d'une part, et réponses de la firme de l'autre. Il peut se résumer par la relation suivante:

$$
\text { PERF }=\mathrm{f}(\text { ENTR, ENVT) }
$$

Pour des environnements différents, des réponses différentes de la PME peuvent conduire au succès, et la recherche rigoureuse de variables d'entreprise significatives sans contrôle de l'environnement sera donc vouée à l'échec.

Peu de recherches font apparaître clairement la nature de la fonction $\mathrm{f}$. La plupart d'entre elles supposent implicitement que les variables de type ENTR sont largement indépendantes de celles de type ENVT, puisqu'elles cherchent des conclusions sur la relation entre PERF et ENTR à partir d'observations d'entreprises appartenant à des environnements différents.

Partant de ce constat, nous avons préféré revenir à une démarche plus modeste s'inspirant de la «quasi-expérience» de Campbell et Stanley (1966) pour compenser le faible nombre d'observations disponibles dans notre champ. En effet, notre travail consiste à identifier les relations entre ENTR et PERF indépendamment des influences des variables d'environnement, en analysant des cas relevant exclusivement de la même industrie et de la même localisation géographique.

Cependant, les relations ainsi mises en évidence ne pourront être valides que dans ce contexte environnemental précis, ce qui n'exclut pas qu'elles puissent avoir une portée plus générale, qui resterait à vérifier dans d'autres contextes. De plus, nous voulons hiérarchiser la liste des facteurs favorisant la performance de l'entreprise, afin d'en dégager les plus importants, ce qui n'apparaît pas clairement dans les recherches empiriques qui traitent de cette question, notamment celles examinées par Aaby et Slater (1989). Le modèle utilisé implicitement par ces auteurs («Strategic Export Model») dans leur revue de littérature serait plutôt du type:

$$
\text { PERF }=\mathrm{f} 1(\text { ENTR })+\mathrm{f} 2(\text { ENVT })
$$

Les recherches ainsi analysées s'intéressent, pour la plupart, uniquement à la description de la fonction $\mathrm{f} 1$. Se distinguant les unes des autres par la mesure de la variable PERF ou par la mise en évidence des relations entre PERF et les variables de type ENTR, elles présentent cependant un intérêt capital pour notre travail: celui de permettre d'énumérer de façon quasi exhaustive tous les facteurs possibles qui peuvent influer sur le niveau de performance de l'entreprise à l'exportation. 


\subsection{Classification des facteurs explicatifs}

La synthèse réalisée par Aaby et Slater (1989) de 55 recherches empiriques menées sur des contextes géographiques aussi différents que les États-Unis, le Canada, la Norvège, le Pérou, la Turquie, l'Allemagne de l'Ouest et la GrandeBretagne fait état de trois catégories de facteurs : les caractéristiques de l'entreprise, ses compétences et les facteurs liés à sa stratégie.

Nous avons retenu cette classification, mais en mettant mieux en évidence les facteurs propres aux PMI et en y intégrant les résultats de Reffait et Roux (1981) qui proposent six catégories de facteurs influençant la performance: les caractéristiques générales de l'entreprise, les caractéristiques individuelles des dirigeants «export», le comportement commercial de l'entreprise, son système d'information «export», son système d'organisation «export» et sa politique commerciale.

Nous rajoutons enfin un facteur considéré par Giordano (1984) comme une condition indispensable de réussite: le fait que la décision d'exporter s'inscrive dans une démarche stratégique globale et ne doive pas être seulement le «résultat d'opportunités passagères », ce qui permet d'obtenir les quatre groupes de facteurs suivants :

- les caractéristiques de la direction de la PMI;

- les caractéristiques générales de l'entreprise;

- les compétences de l'entreprise;

- les facteurs liés à la stratégie de l'entreprise.

Les caractéristiques de la direction de la PMI sont d'abord des caractéristiques individuelles des dirigeants : leur formation préalable, leur degré d'expérience internationale qui comprend notamment l'effet d'apprentissage dans l'activité d'exportation et le statut du responsable «export» de l'entreprisefonction spécifique ou occupée par le directeur commercial ou le directeur général.

Il s'agit ensuite de l'ambition de l'équipe dirigeante de l'entreprise : toutes les recherches revues par Aaby et Slater concluent à l'existence d'une liaison positive entre la performance à l'export et le degré d'ambition exprimé dans l'existence d'une vision stratégique internationale pour la PMI, dans la fixation des objectifs à l'exportation, dans la motivation des dirigeants et leur degré de persévérance dans le temps.

Enfin, les principaux déterminants du succès concernent l'attitude des dirigeants à l'égard du risque, ainsi que leur perception des débouchés potentiels à l'exportation, des occasions à saisir sur le marché local et de leur capacité à régler les problèmes liés à la distribution, au SAV, aux approvisionnements, à la maîtrise des coûts et aux possibilités de production. 
Les caractéristiques générales de l'entreprise sont au nombre de trois. La taille de l'entreprise est un facteur dont la liaison avec la performance à l'export est assez controversée. Les études s'opposent sur la qualité de cette liaison: positive (Christensen et al., 1987), négative (Cooper et Kleinschmidt, 1985) ou inexistante (Roncin, 1982 ; Joffre, 1984 et 1986; Diamantopoulos et Inglis, 1988). Ce facteur est inclus dans notre liste à des fins de contrôle.

Les autres caractéristiques retenues sont l'ancienneté de l'expérience d'exportation et la nature économique du secteur d'activité qui permet de discriminer les performances à l'exportation (Roncin, 1978).

Les compétences de l'entreprise sont les savoir-faire particuliers détenus par l'entreprise qui lui confèrent un avantage par rapport à la concurrence sur les marchés visés.

Ainsi, une avance technologique permet d'expliquer une meilleure performance. Cependant, si les marchés cibles sont situés dans les pays en développement, ce facteur peut être supplanté par un avantage de coût.

L'existence d'une organisation «export» au sein de l'entreprise, permettant d'avoir une approche claire du marché, de l'explorer, de l'analyser systématiquement et de planifier correctement l'activité d'exportation, est un facteur déterminant (Burton et Schlegelmilch, 1987).

Les autres facteurs retenus sont des compétences en management (système de contrôle pour maîtriser la performance, décentralisation de la décision), en contrôle de qualité et en communication, notamment à travers la maîtrise de l'anglais, langue essentielle pour les relations internationales.

Quant aux facteurs liés à la stratégie de l'entreprise, rappelons ici le facteur clé décrit par Giordano (1984) concernant l'intégration de l'activité internationale dans une démarche stratégique globale ainsi que le choix du marché en termes de niveau de pouvoir d'achat (Denis et Depelteau, 1985) et d'étendue géographique - marché mondial ou régional. Enfin, les différents éléments du marketing-mix (politiques de produit, de prix, de promotion et de distribution) constituent autant de facteurs explicatifs importants de la performance dans l'export (Malette et al., 1988; Denis, 1990).

La liste qui vient d'être décrite présente l'avantage de couvrir un champ large de déterminants. De plus, elle montre qu'il n'y a pas de formule claire pour réussir dans l'export, du moins si l'on en croit les résultats des recherches empiriques citées ci-dessus.

Cependant, le caractère général de cette liste, ainsi que les difficultés rencontrées par les chercheurs pour hiérarchiser ces facteurs explicatifs restreignent considérablement son opérationnalité. En choisissant de nous limiter 
à un contexte précis, nous pensons pouvoir approfondir, à partir de situations concrètes, la nature de la relation entre la performance dans l'export et ses déterminants; nous présentons maintenant la méthode utilisée pour cela.

\section{Méthode utilisée}

Nous avons indiqué ci-dessus (à la sous-section 1.1.) l'intérêt qu'il pouvait y avoir à figer le champ d'application de cette recherche; le choix d'une localisation géographique, l'île de la Réunion, et d'une industrie, celle des liqueurs et punchs, découle de deux constatations:

1. D'une part, nous pouvions bénéficier d'un accès relativement facile aux informations, concernant les trois principales entreprises du secteur ayant eu ou réalisant encore actuellement une activité internationale; de plus, à cause du faible nombre de cas, il était plus facile d'obtenir une quantité d'informations plus grande, donc de mieux décrire les causes réelles.

2. D'autre part, sur les trois cas en présence, deux avaient conduit à un échec (arrêt de l'activité d'exportation) et un représentait une situation de réussite (augmentation continue de la part relative des exportations dans les ventes totales). L'opposition des situations permettait ainsi de mettre plus facilement en évidence les variables individuelles et d'entreprise ayant agi.

Notre travail a donc consisté dans une première phase à décrire ces cas. Dans une deuxième, le recours à des experts a permis d'obtenir l'identification qualifiée et la hiérarchisation des facteurs explicatifs du succès ou de l'échec.

\subsection{Phase 1: la collecte des informations}

Les informations de contexte recueillies émanent de sources publiques telles que les rapports d'activité de l'IEDOM (Institut d'émission des départements d'outre-mer), de la Direction des douanes, de l'INSEE ou de la Chambre de commerce et d'industrie de la Réunion.

La description des cas - dont un résumé est présenté dans la section suivante - s'est faite à partir de plusieurs entretiens successifs avec le chef d'entreprise et, le cas échéant, son directeur «export». Ces entretiens ont eu pour but de recueillir des informations sur:

- les caractéristiques de l'entreprise: les faits marquants de son histoire, ses objectifs, son environnement concurrentiel, ses savoir-faire et moyens ; 
- la description factuelle de son expérience d'activité internationale : les marchés visés, les objectifs fixés, la stratégie adoptée, les politiques et moyens mis en œuvre, les problèmes rencontrés et les solutions développées, enfin, les résultats obtenus.

\subsection{Phase 2: l'expertise des cas}

Notre démarche est inspirée des principes de la méthode Delphi (Bordeleau, 1987). La mise en évidence des facteurs explicatifs de la performance à l'exportation s'est faite en ayant recours à des informateurs clés, à des analystes privilégiés des expériences de développement international retenues dans notre travail. La pertinence de cette méthode repose très largement sur le choix de ces experts et sur leur mode d'intervention.

Le choix des experts s'est effectué sur la base de trois critères :

- un degré élevé de compétence dans le domaine du développement international associé à une bonne connaissance de la Réunion ;

- un savoir-faire reconnu dans le management de l'entreprise, soit directement en tant que chef d'entreprise, soit indirectement en occupant une fonction de conseil d'entreprise;

- une capacité de jugement, d'analyse et de synthèse reconnue du fait de la nature des fonctions occupées.

L'application de ces critères a permis de sélectionner quatre experts dénommés pour des raisons de confidentialité E1, E2, E3, E4. Le faible nombre d'experts n'affecte nullement la précision des résultats (Brockhoff, 1975). Il a de plus permis, d'une part, de diminuer considérablement le coût d'obtention des résultats - aucun abandon, contacts directs, délais de réponse rapides - et, d'autre part, de favoriser l'explicitation approfondie des opinions émises.

La confidentialité de l'identité des experts a été totale, y compris entre les experts eux-mêmes, chacun ignorant aussi bien le nombre que l'identité des autres experts.

Le mode d'intervention des experts s'est déroulé en trois étapes, correspondant à trois entretiens individuels successifs avec chaque expert.

Dans la première étape, nous avons recueilli l'explication spontanée des trois situations étudiées, sur la base de leurs propres informations et de celles décrites par nous dans les cas. Ce premier entretien s'est achevé par l'identification des facteurs explicatifs de l'échec ou de la réussite et par une notation de chaque facteur sur une échelle variant de 0 à 5 en fonction de l'importance de sa contribution à l'explication. Le danger d'une telle démarche réside dans le fait que chacun ne parle que de ce qu'il connaît, et dans ses propres termes. 
La deuxième étape a eu pour but de corriger cet inconvénient. Le deuxième entretien a commencé par un travail d'explicitation de chaque facteur, soit par l'expert lui-même, soit par nous afin que l'expert en ait une bonne compréhension, qu'elle soit également partagée par les autres et que la notation se fasse en pleine connaissance de cause. Cette démarche a également permis de regrouper des facteurs formulés différemment, mais très proches dans leur signification. Nous avons ensuite procédé à la confirmation des notes précédentes ou à la notation des facteurs oubliés.

Enfin, chaque expert a pris connaissance, d'une part, des facteurs identifiés et des notes attribuées par les autres experts et, d'autre part, de la liste des facteurs issus de la littérature. Il a pu, par cette confrontation, compléter sa propre liste.

La troisième étape a permis d'aboutir à la sélection des principaux facteurs explicatifs et à leur hiérarchisation. La notation de 0 à 5 avait pour inconvénient de ne pas mettre suffisamment en évidence l'écart d'importance existant entre les facteurs. C'est pour minimiser cet inconvénient que nous avons demandé à chaque expert de répartir un quota de 100 points entre les facteurs les plus contributifs, d'après lui, à l'explication des performances à l'exportation respectives des PMI étudiées. Enfin, nous avons effectué la sommation des points pour tous les experts et procédé au classement hiérarchique des facteurs retenus; ce qui a donné les résultats suivants.

\section{Analyse des résultats}

Pour faciliter la compréhension des listes de facteurs qui suivent, nous présentons d'abord un résumé des trois cas. Nous analyserons ensuite les différences observées dans l'identification des facteurs au fur et à mesure du déroulement de la méthode.

\subsection{Résumé des cas}

L'activité «punchs et liqueurs exotiques » est le fait de trois acteurs économiques locaux : les distilleries DIST-A, DIST-B et DIST-C, désignées ainsi pour des raisons de confidentialité. Ces trois distilleries occupent chacune un créneau du marché local en proposant des produits de gamme courante. Leurs résultats sont bénéficiaires, du fait essentiellement de l'existence de protections soit naturelles (goût spécifique des Réunionnais, attachement historique au punch local), soit réglementaires (taux d'octroi de mer de plus de $50 \%$ appliqué aux importations de boissons alcoolisées, taxes variant du simple au triple selon que les produits sont fabriqués localement ou importés). 
Toutes trois ont également une activité à l'exportation, mais ayant produit des résultats très divers. Le tableau 1 récapitule les caractéristiques de l'activité d'exportation des trois entreprises.

DIST-A et DIST-B, deux vieilles maisons créoles, ont connu des échecs cuisants dans des expériences d'exportation et les ont quasiment abandonnées. Ces deux entreprises ont apparemment procédé de la même manière dans l'attaque des marchés:

- amélioration de la qualité des punchs traditionnels et de la capacité de livraison par des investissements techniques;

- attaque du marché français métropolitain en s'appuyant sur des accords de partenariat passés avec des grossistes-distributeurs afin de toucher les créneaux de grande consommation (via la grande distribution).

Après des investissements parfois élevés pour leur taille, tant techniques que commerciaux, et des frais considérables, les objectifs ont été atteints à hauteur de $10 \%$ seulement. Le retrait dans les deux cas a été la solution adoptée.

DIST-C présente un parcours pour l'instant nettement différent. Créée en 1990, elle affiche des résultats commerciaux assez remarquables, sanctionnant une démarche nouvelle par rapport à celle des deux entreprises précédentes :

- une politique d'innovation systématique centrée sur l'emballage et sur la nature des produits;

- des marques nouvelles suggestives;

- l'attaque de marchés internationaux, autres que métropolitain, rendue possible grâce à l'obtention de prix internationaux : Oscars français, européen et mondial de l'emballage en 1990, 14th International Food Award en 1991, $1^{\text {er }}$ Prix international de l'innovation au SIAAL 1992 et Oscar mondial de l'emballage en 1993.

La réussite commerciale de l'entreprise se matérialise par la présence actuelle de ses produits dans 21 pays, un doublement de chiffre d'affaires export chaque année et des commandes en croissance. En revanche, sa situation financière est difficile, à cause d'une insuffisance chronique de ressources stables.

\subsection{Les facteurs explicatifs identifiés par les experts}

Deux critères ont été utilisés pour analyser les résultats obtenus: le degré d'importance du facteur et le niveau de consensus entre experts. Un facteur est considéré comme important dans l'explication de la performance dans l'export lorsque la moyenne arithmétique des notes qui lui ont été attribuées par les experts est au moins de 3,5. Le degré de consensus est considéré comme élevé lorsque le coefficient de variation associé au facteur, défini comme le rapport entre l'écart type et la moyenne, est inférieur ou égal à 0,3 . 
Nous avons ainsi pu regrouper les facteurs explicatifs en quatre catégories (tableau 2):

- degré d'importance élevé, niveau de consensus élevé : catégorie 1 ;

- degré d'importance moyenne ou faible, niveau de consensus élevé: catégorie 2 ;

- degré d'importance élevé, niveau de consensus faible : catégorie 3 ;

- degré d'importance moyenne ou faible, niveau de consensus faible: catégorie 4.

Catégorie 1 : importance élevée, fort consensus. Les premiers facteurs explicatifs ayant reçu une forte note relèvent du produit. L'adaptation du produit proposé sur le marché intérieur à la spécificité du marché d'exportation prend des formes diversifiées : la nécessité apparaît d'adapter la composition, la présentation du produit, mais aussi de procéder à des ajustements en termes de goûts, de normes, de conditionnement, d'étiquetage. Le produit doit en outre présenter un aspect différenciateur : l'originalité permet à la petite entreprise de se distinguer de ses concurrents dont la notoriété et la place sur le marché visé sont déjà bien établies.

L'état d'esprit du dirigeant de l'entreprise en matière d'exportation constitue un autre facteur clé : sa capacité à prendre en considération les besoins de ses clients, sa prise de conscience de la nécessité d'adapter le produit au marché international, même si celui-ci a particulièrement bien réussi sur le marché local, sa capacité à mettre en évidence un avantage compétitif, à exploiter l'originalité du produit (la capacité créative du chef d'entreprise) sont autant de facteurs qui traduisent cet état d'esprit.

Les experts citent encore la persévérance : la réussite à l'exportation demande du temps. Plus globalement, elle repose sur la capacité de l'entreprise à élaborer une stratégie et à assurer la cohérence des politiques retenues avec cette stratégie.

Le choix du pays cible est également cité comme un facteur clé de réussite.

L'existence d'une organisation «export» à l'intérieur de la petite entreprise est présentée comme une nécessité : elle permet une bonne connaissance du marché cible par la collecte régulière d'informations; elle assure une efficacité plus grande des moyens de prospection, à condition qu'un budget minimum y soit affecté; elle instaure enfin un suivi continuel des contacts sur le terrain.

Catégorie 2: importance moyenne ou faible, fort consensus. Les experts sont assez unanimes pour cette catégorie de facteurs, même s'ils leur accordent une importance moindre, par rapport au groupe précédent (tableau 2). 
Catégorie 3 : importance élevée, faible consensus. Le degré de professionnalisme, seul facteur de cette catégorie, a été jugé important par trois des quatre experts; la position du quatrième expert, maintenue après confrontation des opinions, crée une déviation par rapport aux trois autres. C'est le comportement du chef d'entreprise qui est ici visé: son sérieux, sa capacité à tenir les délais, son honnêteté, l'image individuelle qu'il véhicule.

Catégorie 4: importance moyenne ou faible, faible consensus. Ces facteurs ont été cités par les experts sans que, cependant, l'on puisse aboutir au consensus.

- La capacité de négociation du chef d'entreprise.

- Le degré d'ouverture du chef d'entreprise aux activités internationales: par exemple, devant une perte de marché local, le dirigeant qui réagirait en demandant des barrières tarifaires ou réglementaires supplémentaires est considéré comme ayant un faible degré d'ouverture par rapport à celui qui chercherait à la compenser par une activité d'attaque de marchés externes.

- Le degré d'indépendance par rapport au passé: la capacité de recul par rapport au passé est aussi l'expression du degré d'ouverture du chef d'entreprise. D'après les experts, le poids du passé trop présent dans le cas DIST-A, lequel bénéficiait d'une position forte sur le marché local, montre la nécessité d'une certaine modestie, d'une sorte d'humilité à l'égard des opérations de commerce international.

- La qualité de l'investissement relationnel, c'est-à-dire la connaissance des hommes clés dans le pays cible.

- La maîtrise de la relation avec les acheteurs, illustrée dans les expériences de DIST-A et de DIST-B, dont l'échec est en partie expliqué par une maîtrise faible de ses intermédiaires.

- La nécessité de délais d'exécution courts surtout pour des petites entreprises géographiquement éloignées de leur clientèle a été soulignée avec force.

- La rigueur de gestion.

- Un budget de promotion élevé.

- L'adhésion du personnel à l'activité d'exportation de l'entreprise, qui a été mentionnée par deux des quatre experts. Ceux-ci estiment que le rôle du personnel dans la réalisation des projets et dans l'exécution des commandes sera d'autant mieux tenu que chacun aura pris conscience de l'enjeu de l'activité internationale pour l'entreprise. 
- L'adossement à un marché intérieur: ce facteur exprime l'idée qu'une position solide sur le marché local doit d'abord être recherchée avant d'envisager l'attaque des marchés internationaux, ce qui permettra à l'entreprise d'engranger les ressources financières nécessaires.

- L'image de la Réunion, cependant, encore inexistante sur les marchés internationaux : la région devrait se construire une image positive, en informant les acteurs internationaux sur la capacité de ses entreprises à faire des produits de qualité, à respecter des normes internationales, à disposer d'outils performants de production, à bénéficier de conditions modernes de fonctionnement, de la fiabilité des télécommunications, d'un réseau bancaire et d'un appareil administratif efficaces.

TABleau 1

Caractéristiques de l'activité d'exportation des trois entreprises

\begin{tabular}{|c|c|c|c|}
\hline & DIST-A & DIST-B & DIST-C \\
\hline MARCHÉ VISÉ & $\begin{array}{l}\text { France } \\
\text { métropolitaine }\end{array}$ & $\begin{array}{l}\text { France } \\
\text { métropolitaine }\end{array}$ & $\begin{array}{l}\text { Pays à fort } \\
\text { pouvoir d'achat }\end{array}$ \\
\hline $\begin{array}{l}\text { POSITIONNEMENT } \\
\text { OBJECTIFS }\end{array}$ & $\begin{array}{l}\text { Marché des punchs } \\
\text { Part de marché } \\
\text { de } 10 \% \\
\text { Diversification } \\
\text { du risque-pays }\end{array}$ & $\begin{array}{l}\text { Marché des punchs } \\
\text { Élargissement } \\
\text { du marché } \\
\text { Diversification } \\
\text { du risque-pays }\end{array}$ & $\begin{array}{l}\text { Marché du cadeau } \\
\text { Autonomie } \\
\text { de fonctionnement } \\
\text { Survie }\end{array}$ \\
\hline STRATÉGIE & $\begin{array}{l}\text { Attaque d'un marché } \\
\text { de volume } \\
\text { Différenciation }\end{array}$ & $\begin{array}{l}\text { Attaque d'un marché } \\
\text { de volume } \\
\text { Différenciation }\end{array}$ & $\begin{array}{l}\text { Vente d'un produit } \\
\text { haut de gamme }\end{array}$ \\
\hline $\begin{array}{l}\text { POLITIQUE } \\
\text { DE PRODUIT }\end{array}$ & $\begin{array}{l}\text { Modification } \\
\text { de l'étiquette, } \\
\text { de l'emballage }\end{array}$ & $\begin{array}{l}\text { Aucune adaptation } \\
\text { Le produit exporté } \\
\text { est le même que } \\
\text { le produit local }\end{array}$ & $\begin{array}{l}\text { Produit de qualité } \\
\text { artisanale } \\
\text { Emballage } \\
\text { très original } \\
\text { (forme graphisme) }\end{array}$ \\
\hline $\begin{array}{l}\text { POLITIQUE } \\
\text { DE PRIX }\end{array}$ & $\begin{array}{l}\text { Prix de } 25 \% \\
\text { supérieur à ceux } \\
\text { de la concurrence } \\
\text { métropolitaine }\end{array}$ & Aucune & $\begin{array}{l}\text { Prix de } 20 \% \\
\text { supérieur à ceux } \\
\text { de la concurrence }\end{array}$ \\
\hline $\begin{array}{l}\text { POLITIQUE } \\
\text { DE DISTRIBUTION }\end{array}$ & $\begin{array}{l}\text { Revendeur } \\
\text { métropolitain } \\
\text { Grande distribution }\end{array}$ & Revendeurs & $\begin{array}{l}\text { Épiceries fines } \\
\text { Boutiques } \\
\text { hors taxes }\end{array}$ \\
\hline $\begin{array}{l}\text { POLITIQUE } \\
\text { DE PROMOTION }\end{array}$ & $\begin{array}{l}\text { Animation } \\
\text { sur lieux de vente }\end{array}$ & $\begin{array}{l}\text { Participation } \\
\text { épisodique } \\
\text { aux salons }\end{array}$ & $\begin{array}{l}\text { Participation } \\
\text { systématique } \\
\text { aux salons } \\
\text { Prospections } \\
\text { dans les pays cibles }\end{array}$ \\
\hline
\end{tabular}


Tableau 2

Grille récapitulative des facteurs explicatifs de succès après explicitation, confrontation et regroupements

Facteurs cités

E1

E2

E3

E4

M

CV

(M : note moyenne

CV : coefficient de variation)

Catégorie 1: importance élevée, fort consensus

Adaptation du produit au marché

Originalité du produit

Présentation du produit

Cohérence entre politiques et stratégie

État d'esprit «marketing»

Capacité créative

Avantage compétitif

Persévérance dans le temps

Choix du pays cible

Suivi continuel des contacts

Connaissance du marché

Budget de prospection

$\begin{array}{llllll}5 & 5 & 4 & 5 & 4,7 & 0,09 \\ 5 & 4 & 2 & 5 & 4 & 0,3 \\ 5 & 3 & 3,5 & 4 & 3,8 & 0,28 \\ 3,5 & 5 & 4 & 4 & 4,1 & 0,13 \\ 4 & 3 & 4 & 5 & 4 & 0,17 \\ 4 & 5 & 3 & 3 & 3,7 & 0,22 \\ 3,5 & 3 & 4 & 4 & 3,6 & 0,11 \\ 3 & 3 & 3 & 5 & 3,5 & 0,25 \\ 4 & 4 & 2 & 4 & 3,5 & 0,25 \\ 4 & 4 & 4 & 5 & 4,2 & 0,1 \\ 4 & 3 & 4 & 4 & 3,7 & 0,11 \\ 4 & 3 & 3 & 4 & 3,5 & 0,14\end{array}$

Catégorie 2: importance moyenne ou faible, fort consensus

Accès à l'information

Choix du réseau de distribution

Choix des partenaires

Structure organisationnelle

Notoriété de l'entreprise

Procédures du commerce international

Capacités financières

$\begin{array}{llllll}3 & 3 & 3 & 3 & 3 & 0 \\ 4 & 3 & 3 & 3 & 3,2 & 0,13 \\ 3,5 & 3 & 3 & 3 & 3,1 & 0,07 \\ 3,5 & 3 & 2 & 3 & 2,9 & 0,19 \\ 2 & 3 & 2 & 2 & 2,2 & 0,19 \\ 2 & 3 & 3 & 3 & 2,7 & 0,16 \\ 2 & 2 & 2 & 3 & 2,2 & 0,19\end{array}$

Catégorie 3: importance élevée, faible consensus

Degré de professionnalisme

$\begin{array}{llllll}0 & 4 & 5 & 5 & 3,5 & 0,59\end{array}$

Catégorie 4 : importance moyenne ou faible, faible consensus

Capacité de négociation

Ouverture à l'international

Degré d'indépendance au passé (\%)

Qualité de l'investissement relationnel

Maîtrise des relations avec acheteurs

Délais d'exécution

Rigueur de gestion

Budget de promotion

Adhésion du personnel à l'activité internationale

Adossement à un marché intérieur

Image de la Réunion

\begin{tabular}{llllll}
3 & 4 & 2 & 3 & 3 & 0,24 \\
0 & 5 & 4 & 4 & 3,2 & 0,59 \\
0 & 5 & 4 & 4 & 3,2 & 0,59 \\
0 & 3 & 4 & 4 & 2,7 & 0,6 \\
4 & 4 & 2 & 1 & 2,7 & 0,47 \\
2 & 2 & 4 & 4 & 3 & 0,33 \\
3 & 3 & 2 & 5 & 3,2 & 0,34 \\
0 & 2 & 3 & 4 & 2,2 & 0,66 \\
& & & & & \\
1 & 4 & 2 & 4 & 2,7 & 0,47 \\
2 & 0 & 2 & 0 & 1 & 1 \\
0 & 0 & 0 & 3 & 0,7 & 1,73 \\
\hline
\end{tabular}




\subsection{Les facteurs issus de la littérature}

D'après les experts, certains facteurs issus de la littérature s'appliquent, d'autres ne s'appliquent pas au contexte de la Réunion:

Facteurs ne s'appliquant pas au contexte étudié. Les experts dans un large consensus ont considéré que quatre facteurs ne s'appliquent pas au contexte étudié.

1. La nécessité pour les dirigeants de l'entreprise de détenir une expérience internationale préalable; le handicap des entreprises réunionnaises dans ce domaine peut, selon les experts, être largement compensé par leur créativité et leur volonté de réussir sur les marchés extérieurs.

2. L'ancienneté de l'expérience de l'entreprise dans l'activité internationale ne leur est pas apparue comme pouvant constituer une faiblesse incontournable.

3. Le facteur «taille » n'a pas été non plus jugé comme déterminant.

4. Les avantages de coût ont été écartés compte tenu des possibilités de compensation par la créativité de l'entreprise et l'originalité de son offre.

Facteurs retenus par les experts. Il s'agit plus d'appellations différentes, mieux adaptées aux opinions émises dans les entretiens que de facteurs foncièrement nouveaux.

1. Les systèmes de management: ce facteur recoupe d'une certaine façon la rigueur de gestion, déjà citée par les experts. La littérature précise d'autres éléments tels le contrôle de gestion, la décentralisation de la prise de décision, les aptitudes au management renforcées par des formations continues.

2. L'intégration de l'activité internationale dans une démarche stratégique globale : ce facteur est important; il intervient en complément de tout ce qui avait été souligné par les experts concernant la stratégie de la petite entreprise sur les marchés internationaux. Il est ici beaucoup plus précis, dans la mesure où il s'agit pour l'entreprise de concevoir son activité internationale comme une étape dans sa stratégie globale de développement.

3. Le choix de la dimension mondiale du marché plutôt que le choix de sa dimension régionale est retenu comme favorisant la réussite à l'exportation. 
4. La capacité de communication: elle comprend la maîtrise des langues étrangères et, plus globalement, la capacité de la petite entreprise à bien communiquer avec ses clients sur les marchés ciblés.

5. La perception favorable de la capacité de l'entreprise à maîtriser ses coûts : plus le chef d'entreprise perçoit favorablement sa capacité à faire face aux coûts supplémentaires liés à l'activité internationale, plus la performance à l'exportation aura tendance à être élevée.

\subsection{Liste hiérarchisée finale des facteurs explicatifs dominants}

Cette liste est donnée au tableau 3. Dans une première étape, les experts ont noté sur une échelle allant de 0 à 5 , les facteurs explicatifs qu'ils avaient mis en évidence à l'écoute des cas; il en est résulté le classement qui a été décrit plus haut. En vue de contrôler la stabilité des opinions émises et de bien vérifier la contribution respective des facteurs, nous avons demandé aux experts de répartir un quota de 100 points entre les facteurs considérés par eux comme les plus importants, en complétant leur liste par les facteurs issus de la littérature. Pour cette dernière phase, une rétroaction permettant d'uniformiser les propos tenus n'a pas été effectuée. Il est donc normal que les coefficients de dispersion observés dans les opinions soient plus élevés que pour la liste précédente.

Les résultats, dans leur ensemble, confirment la constance des opinions émises par les experts. Le contrôle s'avère donc positif. Les facteurs se répartissent nettement en deux catégories, selon leur degré d'importance.

Facteurs principaux. Ce nouveau mode de classification met en évidence deux facteurs à forte influence : 1) l'adaptation de l'offre de l'entreprise au marché visé, avec 21 points, et 2) l'ambition du dirigeant de l'entreprise - la vision d'une mission internationale pour l'entreprise, la fixation d'objectifs à l'exportation, la motivation et la persévérance dans le temps - avec 16,5 points.

Ces deux facteurs se détachent nettement des cinq autres dont la moyenne des points se situe entre 7 et 10, avec cependant, une dispersion plus grande des opinions. L'intégration de l'activité internationale dans la démarche stratégique globale arrive au troisième rang avec une moyenne de 10 points. Suivent la capacité créative et de différenciation, le choix du marché et la politique de distribution, l'organisation «export», le degré de professionnalisme.

Facteurs secondaires. Six facteurs présentent une moyenne de points comprise entre 2 et 3,5 :

- perception favorable en matière de maîtrise des coûts ;

- capacité de communication de l'entreprise; 
- systèmes de management ;

- adhésion du personnel à l'activité internationale ;

- montant du budget de prospection;

- état d'esprit «marketing».

Il faut enfin souligner le faible degré de consensus des experts sur la nature de ces facteurs considérés comme de moindre importance dans l'explication de la réussite.

TABLEAU 3

Liste hiérarchisée finale des facteurs explicatifs les plus importants

\begin{tabular}{lcc}
\hline Facteurs retenus & $\begin{array}{c}\text { Moyenne } \\
\text { sur } \mathbf{1 0 0} \text { points }\end{array}$ & $\begin{array}{c}\text { Coefficient } \\
\text { de variation }\end{array}$ \\
\hline 1. Adaptation de l'offre de l'entreprise au marché & 21 & 0,9 \\
2. Ambition du dirigeant de l'entreprise & 16,5 & 0,55 \\
3. Intégration de l'activité internationale & & 1 \\
$\quad$ dans une démarche stratégique globale & 10 & 1,02 \\
4. Capacité de création et de différenciation & 9 & 0,6 \\
5. Choix du marché et distribution & 9 & 0,73 \\
6. Organisation «export» & 8 & 1 \\
7. Degré de professionnalisme & 7,5 & 1,6 \\
8. Perception favorable des problèmes & & 1,6 \\
liés à la maîtrise des coûts & 3,5 & 1,6 \\
9. Capacité de communication & 3,5 & 1,7 \\
10. Systèmes de management & 3,5 & 1,7 \\
11. Adhésion du personnel à l'activité internationale & 3 & 1,75 \\
12. Budget de prospection & 3 & \\
13. État d'esprit «marketing " & 2,5 &
\end{tabular}

\section{Conclusion}

Cette recherche s'inscrit dans une suite de travaux cherchant à préciser la relation entre certaines caractéristiques de la PMI et sa performance à l'exportation: l'analyse de la littérature effectuée a montré que si la liste des variables à considérer semblait assez bien établie, la controverse sur leur relation avec la performance restait très ouverte. 
Les résultats obtenus font apparaître trois groupes de facteurs positifs auxquels nous attribuons des pondérations simples issues de nos scores finaux:

- Les «facteurs de choix stratégique» représentent $40 \%$ : il s'agit de l'adaptation de l'offre de l'entreprise au marché visé, du choix du marché et du mode de distribution, et de l'intégration de l'activité internationale dans une démarche stratégique globale ordonnée.

- Les "caractéristiques du dirigeant de l'entreprise » comptent pour $30 \%$ : on y trouve son ambition d'exporter, son degré de motivation, sa persévérance, son degré de professionnalisme, sa prédisposition à la nécessité d'adapter et de s'adapter, et sa capacité à faire maîtriser les coûts.

- Les facteurs liés au "dispositif opérationnel de l'entreprise » recouvrent les $30 \%$ restants: ce sont la capacité créative de l'équipe, la capacité de différenciation dans toutes les fonctions de l'entreprise, le savoirfaire de la structure «export» en termes de prospection et d'analyse de marché, de planification des opérations et de maîtrise des procédures à l'exportation ; enfin, les capacités de management et de communication interne et externe.

Nous remarquons que seule la seconde catégorie ci-dessus, appelée «caractéristiques du dirigeant», paraît uniformément caractéristique de la PME, tandis que les deux autres semblent fortement dépendantes du contexte stratégique.

Nos résultats sont, dans l'ensemble, conformes à ceux des recherches antérieures. Cependant, ils contredisent certains travaux, notamment ceux de Christensen et al. (1987), Cooper et Kleinschmidt (1985), Roncin (1978; 1982), lesquels affirment l'existence d'une liaison entre la performance à l'exportation et la taille, les avantages de coûts et l'expérience internationale de l'entreprise, autant de facteurs éliminés ici par les experts.

Cette contradiction pourrait s'expliquer par l'importance $(70 \%$ d'après le classement ci-dessus) du rôle du contexte stratégique dans l'identification des facteurs explicatifs de la performance: les cas étudiés relevant tous d'un contexte concurrentiel de spécialisation (au sens du Boston Consulting Group, 1981), il serait alors normal que des facteurs caractéristiques du volume n'aient pas été retenus.

Si cela est vrai, des études où le contexte est soigneusement contrôlé seront nécessaires pour aller au-delà de certains résultats contradictoires concernant l'effet des variables.

L'observation des cas étudiés et les résultats obtenus au cours de cette recherche pourront également intéresser les praticiens et les pouvoirs publics. 
Par exemple, la démarche usuelle des décideurs politiques et financiers locaux en matière d'octroi d'aides et de financement pour l'exportation consiste à privilégier nettement les entreprises perçues comme «ayant déjà fait leurs preuves au pays » et ce, au détriment des porteurs de projets originaux et ambitieux, mais dépourvus d'assises locales.

Or, DIST-A et DIST-B, bien implantées sur le marché réunionnais, étaient a priori les meilleurs candidats à l'exportation dans la continuité de leur domination locale: elles ont connu l'échec, avec un coût pour l'entreprise très élevé dans l'un des cas et ce, malgré des aides publiques considérables et de divers types. En revanche, le cas de DIST-C montre qu'une petite entreprise a pu, à partir de la Réunion, réussir directement à l'exportation sans asseoir au préalable sa position sur le marché intérieur.

Les cas étudiés illustrent bien la difficulté pour une PMI issue d'une économie insulaire, à passer de la position de «substitution aux importations dans un contexte concurrentiel fragmenté » à celle «d'acteur sur les marchés internationaux dans un contexte de spécialisation» (Technoption dans Maynard, 1992): pour le moins, l'évolution n'est pas naturelle.

Si cela est vrai, la réussite de l'économie insulaire à l'exportation peut passer par la sélection directe, rigoureuse et précoce d'un petit nombre de projets par les acteurs financiers publics et privés, sur lesquels seront concentrées les ressources. Mais il leur faudra, pour faire ce choix, utiliser des facteurs clés de succès concrets et préalablement validés, tels que ceux proposés dans cet article.

Bien sûr, les résultats de cette recherche ne permettent pas de conclusion pour un champ étendu de validité. Mais ce champ pourra maintenant être élargi par une recherche utilisant la même méthode et portant sur les deux axes naturels suivants :

- identification des facteurs clés de succès à l'exportation pour des PMI opérant ailleurs, mais dans la même industrie (au sens de Porter) que nos distilleries $\mathrm{A}, \mathrm{B}$ et $\mathrm{C}$;

- transposition de la démarche à d'autres contextes insulaires, pays voisins de la Réunion, ou surtout autres régions périphériques maritimes de la Communauté européenne; cela permettrait de renforcer l'estimation des chances de succès d'opérations de développement par les PME sur lesquelles de gros investissements sont faits vu le retard économique de ces régions.

Ce travail a deux limites principales. La première, largement exposée, concerne le champ d'expérimentation réduit à un territoire et une industrie: il 
n'affecte pas, bien au contraire, la validité des résultats dans ce champ. La seconde limite concerne l'utilisation de techniques de panels d'experts, mises au point en situation de prévision, pour identifier et pondérer des variables explicatives. Nous estimons que cette méthode, avec ses limites, et utilisée avec précaution, est actuellement indispensable à la progression de la recherche en gestion, et complète bien les études du type économétrique sur l'explication de la performance des PMI.

Malgré ces limites, ce travail constitue une première contribution à la création souhaitable d'une bibliothèque d'expériences - à l'instar de travaux récents tels ceux de Joyal et al. (1993) sur les comportements stratégiques des PME exportatrices -, permettant aux chercheurs d'identifier plus rigoureusement les clés de réussite. De plus, elle permettrait aux décideurs une meilleure appréciation concrète de ces mêmes clés.

\section{Bibliographie}

AABY, N. et S. SLATER (1989), «Management influences on export performance: a review of the empirical literature 1978-88 ", International Marketing Review, vol. $6, n^{\circ} 4$, p. $7-26$.

BAudoux, M. et A. RoNCIN (1980), Évaluation et analyse des performances à l'exportation des petites et moyennes entreprises industrielles, Paris, Rapport Cordes, $\mathrm{n}^{\circ} 25-78$.

BAUDOUX, M. et A. RoNCIN (1982), «Évaluation et analyse des performances à l'exportation des petites et moyennes industries ", Recherches Économiques et Sociales, Nouvelle série, $\mathrm{n}^{\circ} 4$, p. 49-79.

BILKEY, W.J. (1978), «An attempted integration of literature on export behavior of firms », Journal of International Business Studies, printemps-été, p. 33-46.

BORDEleAU, Y. (1987), Comprendre et développer les organisations, Montréal, Agence d'Arc inc., p. 246-266.

BOSTON CONSULTING GROUP (1981), «Les systèmes concurrentiels », Perspectives et Stratégie, $\mathrm{n}^{\circ} 121$.

BROCKHOFF, K. (1975), «The performance of forecasting groups in computer dialogue and face-to-face discussion », dans H.A. Lindstone et M. Turoff (éd.), The Delphi Method: Techniques and Applications, Reading, Mass., Addison-Wesley.

BURTON, F.N. et B. SCHLEGELMILCH (1987), «Profile analysis of non-exporters versus exporters grouped by export involvement », International Management Review, vol. $27, \mathrm{n}^{\circ} 1$, p. $38-49$.

Campbell, D.T. et J.C. STANley (1966), Experimental and Quasi-Experimental Designs for Research, Chicago, Rand McNally College Publishing Company. 
CASTALDI, R.M. (1986), «An analysis of the work role of CEO's of small firms", American Journal of Small Business, vol. 11, $\mathrm{n}^{\circ} 1$, p. 53-64.

Christensen, C.H., A. DA Rocha et R.K. GerTner (1987), «An empirical investigation of the factors influencing exporting success of Brasilian firms », Journal of International Business Studies, automne, p. 61-77.

COOPER, R.G. et E.J. KLEINSCHMIDT (1985), «The impact of export strategy on export sales performance», Journal of International Business Studies, printemps, p. 37-55.

DENIS, J. (1990), Une synthèse de la recherche sur le comportement des firmes à l'exportation, Université d'Ottawa, Document de travail, $\mathrm{n}^{\circ}$ 90-15.

Denis, J. et D. DePelteau (1985), «Market knowledge, diversification and export expansion », Journal of International Business Studies, automne, p. 77-89.

DiAmANTOPOULOS, A. et K. INGLIS (1988), «Identifying differences between high - and low - involvement exporters », International Marketing Review, vol. 5, été, p. 52-60.

GioRdano, Y. (1984), «Stratégie d'entreprise et décision d'exporter », Revue Française de Gestion, juin-août, p. 113-119.

GIORDANO, Y. (1985), «Politique à l'exportation: les PMI, nouveaux interlocuteurs », VIIes Journées Nationales des IAE, Lille, avril.

JOFFRE, P. (1984), «Le mythe de la taille dans le comportement international des entreprises petites et moyennes », Analyses de la SEDEIS, vol. 15, mars, p. 86-93.

JOFFRE, P. (1986), «Le rôle de la taille ne doit pas être surestimé », Revue Française de Gestion, vol. 55, p. 68-77.

Johnston, W.J. et M.R. Czinkota (1982), «Managerial motivations as determinants of industrial export behavior», dans Export Management - An International Context, édité par M.R. Czinkota et G. Tesar, New York, p. 55-69.

Joyal, A., P.A. Julien, L. Deshaies et C. Ramangalahy (1993), Typologie des comportements stratégiques des PME exportatrices, Rapport de synthèse de recherche, GREPME, Université du Québec à Trois-Rivières, GREPME.

Julien, P.A. et M. MARChESNAY (1988), La petite entreprise, Paris, Vuibert gestion.

KLEINSCHMIDT, E.J. et R.E. Ross (1984), «Export performance and foreign market information : relationship for small high-technology firms », Journal of Small Business, vol. 2, $\mathrm{n}^{\circ} 2$, p. 8-23.

Malette, H., J.M. Denis et D. BÉliveAu (1988), La performance à l'exportation, une consolidation des évidences empiriques, Université d'Ottawa, Document de travail, $\mathrm{n}^{\circ} 88-28$. 
MAYNARD, B. (1992), Technologie et localisation: extension de l'analyse stratégique de la localisation de valeur ajoutée industrielle au cas de motif dominant technologique, Thèse de doctorat en sciences économiques, Université de Nice.

McNAUGHTON, R.B. (1992), «The export behavior of small canadian manufacturing firms », Professional Geographer, vol. 44, $n^{\circ} 2$, p. 170-180.

MIESENBOCK, K.J. (1988), «Small businesses and exporting: a literature review », International Small Business Management, vol. 6, $\mathrm{n}^{\circ}$ 2, p. 42-61.

PORTER, M.E. (1982), Choix stratégique et concurrence, Paris, Économica.

PrAS, B. et E. Roux (1985), «Forces et faiblesses des dirigeants de PMI», Enseignement et Gestion, ${ }^{\circ} 36$, p. 59-65.

RefFaIt, P. et E. RouX (1981), «Le profil idéal de la PME exportatrice», Revue Française de Gestion, janv.-févr., p. 88-96.

RICOL, R. (1990), Mission PME Europe, Paris, Rapport à Madame E. Cresson, ministre des Affaires européennes, 27 février.

Roncin, A. (1978), «Le rôle des caractéristiques sectorielles dans la détermination des performances à l'exportation », Revue d'Économie Industrielle, $\mathrm{n}^{\circ} 3,1^{\mathrm{er}}$ trimestre.

RonCIN, A. (1982), «L'engagement des PMI dans l'exportation», Économie et Statistiques, $\mathrm{n}^{\circ} 148$, octobre, p. 39-51.

Roux, E. (1991), Les facteurs explicatifs de la décision d'exporter en PMI: rôle de l'attitude du dirigeant envers le risque, Thèse d'État en sciences de gestion, IAE d'Aix-en-Provence.

Wiedersheim-Paul, F., H. Olson et L. Welch (1978), «Pre-export activity : the first step in internationalisation », Journal of International Business Studies, printemps-été, p. 47-56. 